\title{
Osteoporosis in Healthy South Indian Males and the Influence of Life Style Factors and Vitamin D Status on Bone Mineral Density
}

\author{
Sahana Shetty, Nitin Kapoor, Dukhabandhu Naik, \\ Hesarghatta Shyamasunder Asha, Suresh Prabu, Nihal Thomas, \\ Mandalam Subramaniam Seshadri, and Thomas Vizhalil Paul \\ Department of Endocrinology, Diabetes \& Metabolism, Christian Medical College \& Hospital, Vellore 632004, India \\ Correspondence should be addressed to Thomas Vizhalil Paul; thomasvpaul@yahoo.com
}

Received 22 August 2014; Revised 21 October 2014; Accepted 22 October 2014; Published 11 November 2014

Academic Editor: Jun Iwamoto

Copyright (C) 2014 Sahana Shetty et al. This is an open access article distributed under the Creative Commons Attribution License, which permits unrestricted use, distribution, and reproduction in any medium, provided the original work is properly cited.

Objective. To study the prevalence of osteoporosis and vitamin D deficiency in healthy men and to explore the influence of various life style factors on bone mineral density (BMD) and also to look at number of subjects warranting treatment. Methods. Ambulatory south Indian men aged above 50 were recruited by cluster random sampling. The physical activity, risk factors in the FRAX tool, $\mathrm{BMD}$, vitamin D, and PTH were assessed. The number of people needing treatment was calculated, which included subjects with osteoporosis and osteopenia with 10-year probability of major osteoporotic fracture $>20$ percent and hip fracture $>3$ percent in FRAX India. Results. A total of 252 men with a mean age of 58 years were studied. The prevalence of osteoporosis and osteopenia at any one site was $20 \%(50 / 252)$ and $58 \%$, respectively. Vitamin D deficiency $(<20 \mathrm{ng} / \mathrm{dL})$ was seen in $53 \%$. On multiple logistic regression, $\mathrm{BMI}(\mathrm{OR} 0.3 ; P$ value $=0.04)$ and physical activity $(\mathrm{OR} 0.4 ; P$ value $<0.001)$ had protective effect on BMD. Twenty-five percent warranted treatment. Conclusions. A significantly large proportion of south Indian men had osteoporosis and vitamin D deficiency. Further interventional studies are needed to look at reduction in end points like fractures in these subjects.

\section{Introduction}

Osteoporosis in men is now recognized as a major underestimated public health problem [1]. With the gradual increase in life expectancy, advancing age related illnesses are increasing [2]. After the age of fifty, one out of three osteoporotic fractures are seen in men. Furthermore, an in depth understanding of this subject has revealed that about fifty percent of these causes are potentially treatable. Studies have shown that men with osteoporotic fractures have a much higher mortality and morbidity when compared to women [3]. This may add on to the economic burden in a developing country like India, where men may be the only earning members in many families [4].

In addition to genetic determinants, several life-style related factors like physical activity, calcium intake, smoking, alcohol consumption, and vitamin D status may influence the bone mass in men [5]. However, the prevalence and influence of these factors may vary according to ethnicity.

Screening for osteoporosis in men is usually recommended above the age of 70 years $[1,5]$. However, its relevance in relation to the variability in ethnicity requires validation through prospective studies. There are differences in peak bone mass, body frame, and nutrition and life style factors among various populations [1]. There are no clear guidelines available for screening men with osteoporosis among ethnic groups other than the Caucasian population.

There is also a paucity of data with regards to the risk factors that have been mentioned and their influence on bone health in an Indian context. Many studies have shown that a low Bone Mineral Density (BMD) may not be the sole factor which determines the risk of fracture [6]. This led on to developing a web based tool FRAX (Fracture Risk Assessment) to assess the fracture risk which incorporated 
various risk factors like parental hip fracture, smoking, alcohol consumption, past history of fracture, and other factors in addition to femoral neck BMD [7]. This tool calculates a ten-year probability of major osteoporotic fracture and hip fracture.

In this study, we have attempted to look at the prevalence of osteoporosis and vitamin D status in healthy South Indian men and to study the influence of various life style factors on bone mineral density. The number of subjects warranting treatment in these healthy subjects was also computed.

\section{Materials and Methods}

The study was a cross sectional one conducted over a duration period of 1 year. This study was approved by Institutional Review Board.

We conducted a survey of the total number of houses in an urban region of south India. Men above 50 years of age in that locality were recruited by cluster random sampling after obtaining a written informed consent. Men with a history of chronic liver or renal disease, hyperthyroidism, hyperparathyroidism, hypogonadism, and malabsorption and those on medications such as anticonvulsants, antiretrovirals, and antituberculous therapy which will affect the bone health were excluded.

The risk factors which were mentioned in the FRAX tool assessment included age, sex, height, weight, a past history of fragility fracture, parental history of hip fracture, history of smoking or alcohol intake (3 or more units/day), the presence of rheumatoid arthritis, and any other history suggestive of secondary osteoporosis [7]. Physical activity was evaluated using a previously published questionnaire standardized for the Indian population and was categorized with a scoring system of less than 1.4 as sedentary, 1.55 to 1.6 as moderately active, and more than 1.75 as strenuous active [8].

An overnight fasting blood sample was obtained for estimation of serum calcium (8.6-10.2 mg/dL), phosphate (2.5$5 \mathrm{mg} / \mathrm{dL})$, albumin $(3.5-5.0 \mathrm{gm} / \mathrm{dL})$, alkaline phosphatase (40-125 iu/L), creatinine $(0.6-1.2 \mathrm{mg} / \mathrm{dL}), 25$-hydroxy vitamin D $(30-75 \mathrm{ng} / \mathrm{mL})$ hereafter referred to as vitamin $\mathrm{D}$, intact parathyroid hormone (iPTH) $(8-50 \mathrm{pg} / \mathrm{mL})$, and testosterone (300-1000 ng/dL). These reference ranges are as provided by the local laboratory. Vitamin D deficiency was defined as a vitamin $\mathrm{D}$ level of less than $20 \mathrm{ng} / \mathrm{mL}$ and a level less than $10 \mathrm{ng} / \mathrm{mL}$ was considered to indicate severe vitamin $\mathrm{D}$ deficiency [9]. The vitamin $\mathrm{D}$, intact $\mathrm{PTH}$, and testosterone were measured by chemiluminescence method using Immulite analyzer 2000. Other biochemical parameters were measured in a fully automated and computerized microanalyzer (Hitachi Roche Modular P 800 model). The intra-assay and interassay coefficients of variation for these analytes were 1 to $5 \%$.

BMD was assessed using the Hologic DXA QDR 4500 Discovery A machine at the lumbar spine and femoral neck by the same technician. Precision was 2 percent at both themeasured sites (spine and neck of femur). The WHO classification was used for categorization of BMD [10].
Osteoporosis was defined as $T$ score $\leq-2.5$, osteopenia, or low bone mass -1 to -2.5 and normal as $>-1$.

In subjects who were found to have osteopenia, data regarding all risk factors were incorporated into a web based software-Fracture Risk Assessment_FRAX India for calculating the 10-year probability of major osteoporotic fracture and hip fracture. The number of people needing treatment was calculated, which included the total number of subjects with osteoporosis at any site and osteopenia with a 10-year probability of major osteoporotic fracture more than 20 and hip fracture more than 3 as calculated by FRAX India. This was as per the Endocrine Society clinical practice guidelines [11].

2.1. Sample Size Calculation and Statistical Analysis. The prevalence of male osteoporosis above the age of 50 years in published literature was about 6\% [12]. Total number of men in an urban area of Southern India above the age of 50 years was 2120 . The sample size required for a precision of $5 \%$ and a 95 confidence interval and a power of $80 \%$ was 240 . The cluster random sampling was used to recruit subjects for the study. Independent $T$-test was used to compare the means of two continuous variables if they were normally distributed and nonparametric tests were used if their distribution were not normal. Statistical analysis was done using the SPSSv.16.0 software (IBM Corp., USA).

\section{Results}

Two hundred and fifty-two men above the age of 50 years were included in the study. The demographic parameters, biochemistry including vitamin D status, and BMD of the study subjects are summarized in Table 1.

The mean age (SD) of the study subjects was 58 (11.8) years and the mean (SD) BMI was $23.3(4.5) \mathrm{kg} / \mathrm{m}^{2}$. Forty percent $(n=100)$ of men were above the age of 60 years. In relation to physical activity, 77 percent were sedentary with the rest being moderately to strenuously active.

The prevalence of osteoporosis and osteopenia at spine and femoral neck is shown in Figure 1. The osteoporosis was seen in $15 \%$ and $10 \%$ of the subjects at the spine and femoral neck, respectively. A significantly greater proportion of subjects aged above 60 years had osteoporosis at the femoral neck when compared to men below that age $(15.6 \%$ versus $7.5 \%, P=0.01)$. Osteoporosis at the spine was also more commonly seen among subjects aged above the age of 60 years; however, it was not statistically significant.

Overall the prevalence of osteoporosis and osteopenia at any one site was 20\% (50/252) and 58\% (146/252), respectively. However, in men aged above 60 years, the prevalence of osteoporosis was $45 \%$. Vitamin D deficiency $(<20 \mathrm{ng} / \mathrm{dL})$ was seen in 53\% $(n=133)$ and $7 \%$ had severe vitamin $\mathrm{D}$ deficiency $(<10 \mathrm{ng} / \mathrm{dL})$. Eight percent of subjects had biochemical hypogonadism $(<300 \mathrm{ng} / \mathrm{dL})$.

Among the risk factors studied, BMD at the femoral neck was significantly higher in subjects who were physically more active when compared to men who had a sedentary lifestyle (0.811 versus $\left.0.733 \mathrm{gm} / \mathrm{cm}^{2}, P=0.005\right)$. Multiple logistic 
TABLE 1: Demography, biochemistry, and BMD.

\begin{tabular}{lcc}
\hline Variables & $\begin{array}{c}\text { Mean (standard } \\
\text { deviation) }\end{array}$ & Range \\
\hline Age (years) & $58.8(11.8)$ & $51-74$ \\
Height $(\mathrm{cms})$ & $165.4(7.2)$ & $142.2-183.0$ \\
Weight $(\mathrm{kgs})$ & $63.7(14.2)$ & $35-114$ \\
BMI $\left(\mathrm{kg} / \mathrm{m}^{2}\right)$ & $23.3(4.5)$ & $16.2-41.6$ \\
Corrected calcium $(\mathrm{mg} \%)$ & $8.82(0.43)$ & $8.1-10.2$ \\
Phosphorus $(\mathrm{mg} \%)$ & $3.9(0.5)$ & $2.1-5.1$ \\
Creatinine $(\mathrm{mg} \%)$ & $0.96(0.12)$ & $0.7-1.3$ \\
Vitamin D $(\mathrm{ng} / \mathrm{mL})$ & $20.4(8.3)$ & $4-58$ \\
Alkaline phosphatase $(\mathrm{U} / \mathrm{L})$ & $73.5(21.4)$ & $39-167$ \\
Testosterone $\left(\mathrm{ng} / \mathrm{dL}^{2}\right)$ & $620(124)$ & $270-980$ \\
PTH (pg/mL) & $44.5(25.6)$ & $14.5-151.0$ \\
BMD spine $\left(\mathrm{gm} / \mathrm{cm}^{2}\right)$ & $0.943(0.111)$ & $0.912-0.974$ \\
BMD neck $\left(\mathrm{gm} / \mathrm{cm}^{2}\right)$ & $0.761(0.124)$ & $0.733-0.771$ \\
\hline
\end{tabular}

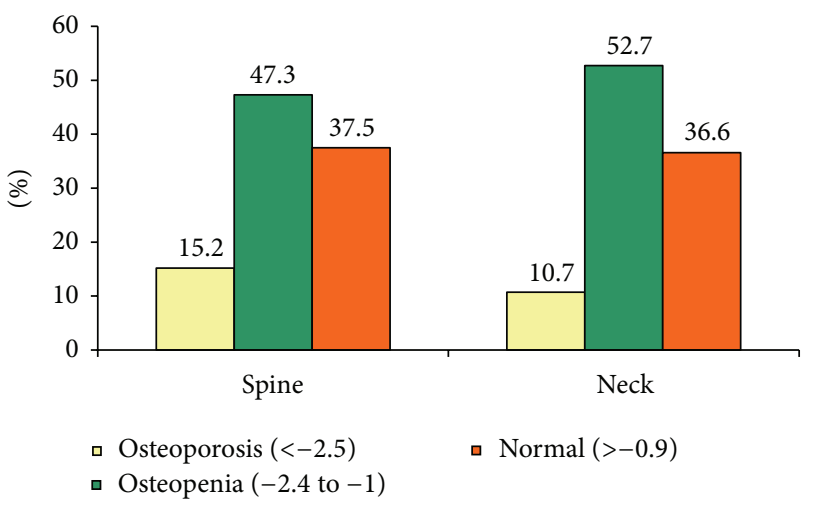

FIGURE 1: Showing categorization of BMD in the study subjects.

regression analysis was performed to assess the impact of various factors that influence bone mineral density at femoral neck in healthy male subjects. These included body mass index, hypogonadism, age, vitamin D deficiency, smoking, alcohol use, and physical activity. We found that body mass index (OR 0.3; CI 0.1 to $0.40 ; P$ value $=0.04$ ) and physical activity (OR 0.4 ; CI 0.12 to $0.9 ; P$ value $<0.0001$ ) had a statistically significant protective effect on bone mineral density.

Amongst the subjects who were having osteopenia at any one site $(n=146) 13$ men had a greater than $3 \%$ probable risk of hip fracture; however, none of these subjects had more than a 20 percent risk of major osteoporotic fracture calculated by FRAX India.

Therefore, out of the total 256 subjects, 63 warranted treatment (50 had osteoporosis plus 13 osteopenics with a more than 3\% probable risk of hip fracture). The mean (SD) age of subjects warranting therapy in our study was 67.5 (11.65) years. There was a significant negative correlation between vitamin $\mathrm{D}$ and PTH levels $(r=-0.29, P=0.04)$. However, there is no significant correlation between vitamin
$\mathrm{D}$ and $\mathrm{BMD}$ at any site and also no significant correlation was found between PTH and BMD.

\section{Discussion}

Male osteoporosis is an underreported public health problem. In our study, we attempted to look at the prevalence of osteoporosis and the various risk factors in South Indian healthy men above the age of 50 years.

In this present study, about one-fifth of them had osteoporosis at any one site. Vitamin D deficiency was found in over half of the study population. Men who were physically active or having higher body mass index had a better BMD at femoral neck. As per the FRAX score, about 10 percent of the osteopenic subjects had more than a $3 \%$, tenyear probability of sustaining a hip fracture. Mean age of warranting treatment to prevent fractures was close to the recommendations published in the international guidelines [11]. Treatment was warranted in one out of four healthy subjects who had either osteoporosis or osteopenia with a computed ten-year probability of hip fracture of more than $3 \%$ as per the FRAX score.

4.1. Prevalence of Osteoporosis. In previously published literature, a 9 percent prevalence of osteoporosis has been reported in Northern India [13] and in oriental men [14]. However, in another study at Rochester [15], a 19\% prevalence of osteoporosis has been reported, which bears similarity to our study. The differences in the prevalence that was seen between south Indian and north Indian subjects would have been due to many factors like genetic, nutritional, and other environmental factors. They need to be looked at in further prospective studies.

BMD begins to decline after the third decade and is influenced by genetic and environmental factors. Prepubertal BMD is similar in both sexes. However, the pubertal increase in BMD is more in men when compared to women due to a greater cross sectional area in view of increased periosteal apposition under the influence of androgens [16]. Bone remodeling with aging leads to trabecular thinning in men, whereas trabecular connectivity is lost in women [17]. Decline in BMD in men may begin as early as $30-40$ years. However, the accelerated menopausal bone loss may not be seen. The estimated rate of bone loss with aging in men is about $1 \%$ per year [16].

4.2. Osteoporosis and Risk of Fracture: Morbidity, Mortality, and Economic Burden Associated with Fracture. The most dreaded complication of osteoporosis is hip fracture, which has been reported to be more in men when compared to women [18]. Osteoporosis in men accounts for more than 30$40 \%$ of overall fracture. Fracture in men follows a bimodal presentation with peaks at adolescence and after 60 years [19]. The morbidity and mortality associated with hip fractures in men has been reported as high as $33 \%[18,20]$. Moreover, a three to four times higher mortality rate has been described in men with hip fracture when compared to females [3, 21]. 
The economic burden of osteoporotic fractures is not only borne by the patient but by the country as a whole [22, 23]. The major costs may not only include that of investigations, surgery, or long term management of these patients but also the large number of man hours that is lost by these men after they sustain a fracture. This is particularly relevant in developing countries like India where there is an increase in the aging population [24]. About 25\% of our subjects needed treatment in accordance with endocrine society guidelines [11]. Considering the population of men above 65 years in India (about 27 million) and the available incidence of hip fracture in men above 50 years $(105 / 100,000)$, the magnitude of the problem is huge $[25,26]$. It translates into having about 25,000 hip fractures in elderly men ( $>65$ years) every year and about one-third to one-fourth of them dying in 1 to 2 years. The mere cost of treating each fracture is about 90,000 rupees (about 1500 US dollars) in India showing huge economic burden associated with them. At this point, there is a limited availability of DXA scanners in the country excluding major cities [24]. So, the health care system at the primary level can use FRAX scoring (utilizing BMI without BMD) to plan preventive strategies which in turn will decrease the morbidity, mortality, and economic costs associated with them. However, further studies are needed with such interventions to look at the impact of these in a larger cohort of elderly men. It will enable the national bodies to come up with clinical practice guidelines relevant in an Indian context.

4.3. Vitamin D Deficiency: Its Impact on Bone Health. A high prevalence of vitamin $\mathrm{D}$ deficiency has been reported in various cohorts of Indian population [27]. Vitamin D deficiency can either decrease mineralization or cause secondary hyperparathyroidism or both resulting in a low bone mineral density [28]. A low vitamin D may also cause proximal myopathy predisposing these patients to a fall and subsequent fracture [27].

4.4. Role of Physical Activity and BMI. A positive impact of physical activity on bone mass or BMD demonstrated in our study is probably due to a skeletal response to mechanical strain by stimulating bone formation [29]. Also increase in the muscle strength and neuromuscular function seen secondary to physical activity has been shown to decrease the incidences of fall and a resulting fracture [30]. A reduction in fracture risk has also been reported on follow-up in subjects who were physically active [31].

An increase in body mass index had a significant impact on BMD. Several studies have shown the positive correlation between BMI and BMD. This is explained by a higher gravitational load on the femur neck, increased peak bone mass, and higher circulating estradiol level [28].

\section{Limitations of the Study}

The socioeconomic status, nutritional status, prevalent vertebral fractures, and bone turnover markers in these individuals were not assessed. Also this data has been collected from an urban society in southern India and cannot be generalized to the entire Indian population.

\section{Conclusions}

A significantly larger proportion of otherwise normal healthy men in our community had osteoporosis and vitamin $\mathrm{D}$ deficiency compared to previously published studies. Men with a higher BMI were physically active and had a better BMD. Large scale prospective studies with interventions are needed to look at the reduction in the end points like number of incident fractures and morbidity associated with them.

\section{Conflict of Interests}

The authors declare that there is no conflict of interests regarding the publication of this paper.

\section{Authors' Contributions}

All authors have contributed to data analysis, paper writing, and reviewing and approving the final paper.

\section{References}

[1] P. R. Ebelin, "Osteoporosis in men," The New England Journal of Medicine, vol. 358, pp. 1474-1482, 2008.

[2] J. M. Jacobs, Y. Maaravi, A. Cohen, M. Bursztyn, E. Ein-Mor, and J. Stessman, "Changing profile of health and function from age 70 to 85 years," Gerontology, vol. 58, no. 4, pp. 313-321, 2012.

[3] D. Bliuc, N. D. Nguyen, V. E. Milch, T. V. Nguyen, J. A. Eisman, and J. R. Center, "Mortality risk associated with low-trauma osteoporotic fracture and subsequent fracture in men and women," JAMA-Journal of the American Medical Association, vol. 301, no. 5, pp. 513-521, 2009.

[4] S. S. Vepa, "Gender equity \& human development," The Indian Journal of Medical Research, vol. 126, no. 4, pp. 328-340, 2007.

[5] S. S. Rao, N. Budhwar, and A. Ashfaque, "Osteoporosis in men," American Family Physician, vol. 82, no. 5, pp. 503-508, 2010.

[6] J. A. Kanis, O. Johnell, A. Oden, B. Jonsson, A. Dawson, and W. Dere, "Risk of hip fracture derived from relative risks: an analysis applied to the population of Sweden," Osteoporosis International, vol. 11, no. 2, pp. 120-127, 2000.

[7] J. A. Kanis, O. Johnell, A. Oden, H. Johansson, and E. McCloskey, "FRAX and the assessment of fracture probability in men and women from the UK," Osteoporosis International, vol. 19, no. 4, pp. 385-397, 2008.

[8] A. V. Bharathi, N. Sandhya, and M. Vaz, "The development and characteristics of a physical activity questionnaire for epidemiological studies in urban middle class Indians," Indian Journal of Medical Research, vol. 111, pp. 95-102, 2000.

[9] K. A. Kennel, M. T. Drake, and D. L. Hurley, "Vitamin D deficiency in adults: when to test and how to treat," Mayo Clinic Proceedings, vol. 85, no. 8, pp. 752-758, 2010.

[10] World Health Organization (WHO), "Assessment of fracture risk and its application to screening for postmenopausal osteoporosis: report of a WHO study group," WHO Technical Report 843, WHO, Geneva, Switzerland, 1994.

[11] N. B. Watts, R. A. Adler, J. P. Bilezikian et al., "Osteoporosis in men: an endocrine society clinical practice guideline," The 
Journal of Clinical Endocrinology \& Metabolism, vol. 97, no. 6, pp. 1802-1822, 2012.

[12] L. Gennari and J. P. Bilezikian, "Osteoporosis in men," Endocrinology and Metabolism Clinics of North America, vol. 36, no. 2, pp. 399-419, 2007.

[13] N. K. Agrawal and B. Sharma, "Prevalence of osteoporosis in otherwise healthy Indian males aged 50 years and above," Archives of Osteoporosis, vol. 8, no. 1-2, article no. 116, 2013.

[14] Y. K. Lee, B. H. Yoon, and K. H. Koo, "Epidemiology of osteoporosis and osteoporotic fractures in South Korea," Endocrinology and Metabolism, vol. 28, no. 2, pp. 90-93, 2013.

[15] L. J. Melton III, E. J. Atkinson, M. K. O'Connor, W. M. O’Fallon, and B. L. Riggs, "Bone density and fracture risk in men," Journal of Bone and Mineral Research, vol. 13, no. 12, pp. 1915-1923, 1998.

[16] R. P. Heaney, S. Abrams, B. Dawson-Hughes et al., "Peak bone mass," Osteoporosis International, vol. 11, no. 12, pp. 985-1009, 2000.

[17] B. L. Riggs, L. J. Melton III, R. A. Robb et al., "Population-based study of age and sex differences in bone volumetric density, size, geometry, and structure at different skeletal sites," Journal of Bone and Mineral Research, vol. 19, no. 12, pp. 1945-1954, 2004.

[18] J. R. Center, T. V. Nguyen, D. Schneider, P. N. Sambrook, and J. A. Eisman, "Mortality after all major types of osteoporotic fracture in men and women: an observational study," The Lancet, vol. 353, no. 9156, pp. 878-882, 1999.

[19] T. H. Diamond, S. W. Thornley, R. Sekel et al., "Hip fracture in elderly men: prognostic factors and outcomes," Medical Journal of Australia, vol. 167, no. 8, pp. 412-415, 1997.

[20] G. S. Keene, M. J. Parker, and G. A. Pryor, "Mortality and morbidity after hip fractures," The British Medical Journal, vol. 307, no. 6914, pp. 1248-1250, 1993.

[21] D. Bliuc, N. D. Nguyen, V. E. Milch, T. V. Nguyen, J. A. Eisman, and J. R. Center, "Mortality risk associated with lowtrauma osteoporotic fracture and subsequent fracture in men and women," Journal of the American Medical Association, vol. 301, no. 5, pp. 513-521, 2009.

[22] B. Qu, Y. Ma, M. Yan et al., "The economic burden of fracture patients with osteoporosis in western China," Osteoporosis International, vol. 25, no. 7, pp. 1853-1860, 2014.

[23] E. Hernlund, A. Svedbom, M. Ivergård et al., "Osteoporosis in the European Union: medical management, epidemiology and economic burden: a report prepared in collaboration with the International Osteoporosis Foundation (IOF) and the European Federation of Pharmaceutical Industry Associations (EFPIA)," Archives of Osteoporosis, vol. 8, article 136, 2013.

[24] N. Malhotra and A. Mithal, "Osteoporosis in Indians," Indian Journal of Medical Research, vol. 127, no. 3, pp. 263-268, 2008.

[25] D. K. Dhanwal, R. Siwach, V. Dixit, A. Mithal, K. Jameson, and C. Cooper, "Incidence of hip fracture in Rohtak district, North India," Archives of Osteoporosis, vol. 8, no. 1-2, article 135, 2013.

[26] P. Gupta, K. Mani, S. K. Rai, B. Nongkynrih, and S. K. Gupta, "Functional disability among elderly persons in a rural area of Haryana," Indian Journal of Public Health, vol. 58, no. 1, pp. 1116, 2014.

[27] C. V. Harinarayan and S. R. Joshi, "Vitamin D status in India-its implications and remedial measures," Journal of Association of Physicians of India, vol. 57, pp. 40-48, 2009.

[28] T. V. Paul, N. Thomas, M. S. Seshadri, R. Oommen, A. Jose, and N. V. Mahendri, "Prevalence of osteoporosis in ambulatory postmenopausal women from a semiurban region in southern India: relationship to calcium nutrition and vitamin D status," Endocrine Practice, vol. 14, no. 6, pp. 665-671, 2008.
[29] M. Martyn-St James and S. Carroll, "A meta-analysis of impact exercise on postmenopausal bone loss: the case for mixed loading exercise programmes," British Journal of Sports Medicine, vol. 43, no. 12, pp. 898-908, 2009.

[30] E. W. Gregg, M. A. Pereira, and C. J. Caspersen, "Physical activity, falls, and fractures among older adults: a review of the epidemiologic evidence," Journal of the American Geriatrics Society, vol. 48, no. 8, pp. 883-893, 2000.

[31] M. K. Karlsson, C. Linden, C. Karlsson, O. Johnell, K. Obrant, and E. Seeman, "Exercise during growth and bone mineral density and fractures in old age," The Lancet, vol. 355, no. 9202, pp. 469-470, 2000. 


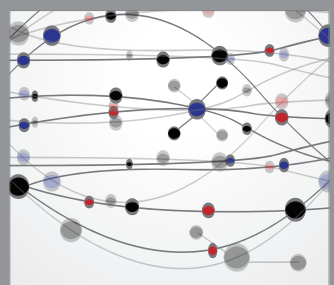

The Scientific World Journal
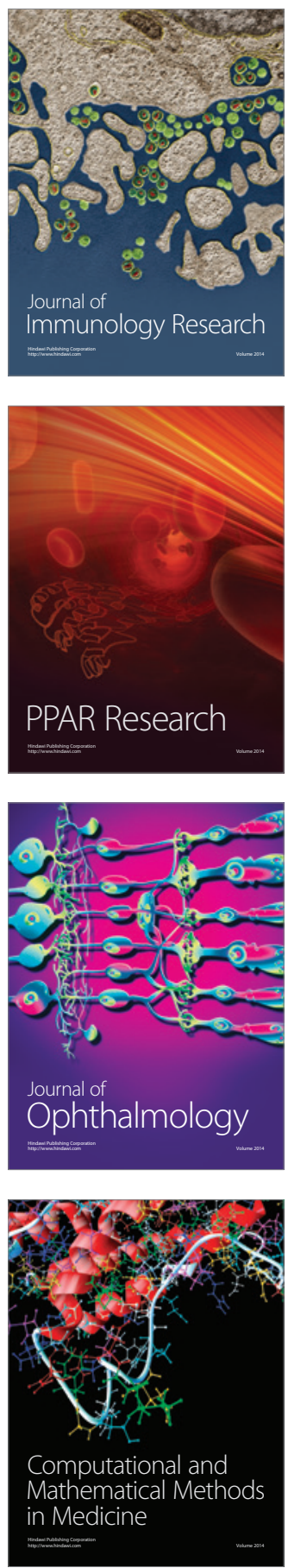

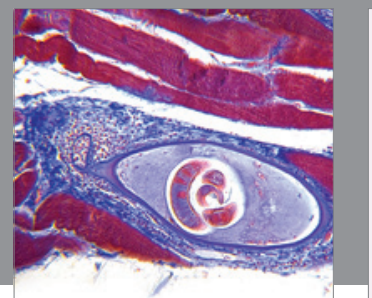

Gastroenterology

Research and Practice
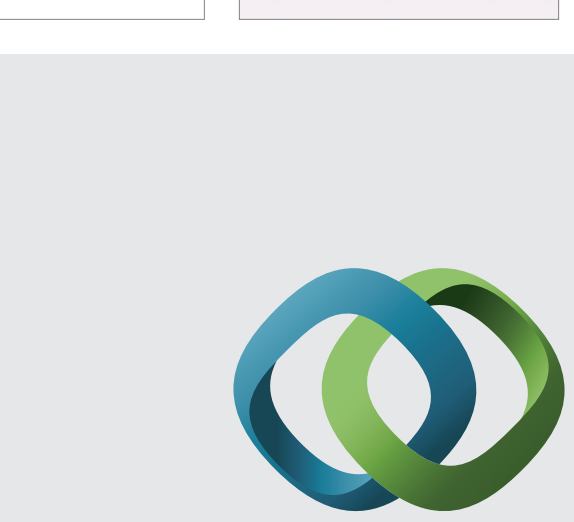

\section{Hindawi}

Submit your manuscripts at

http://www.hindawi.com
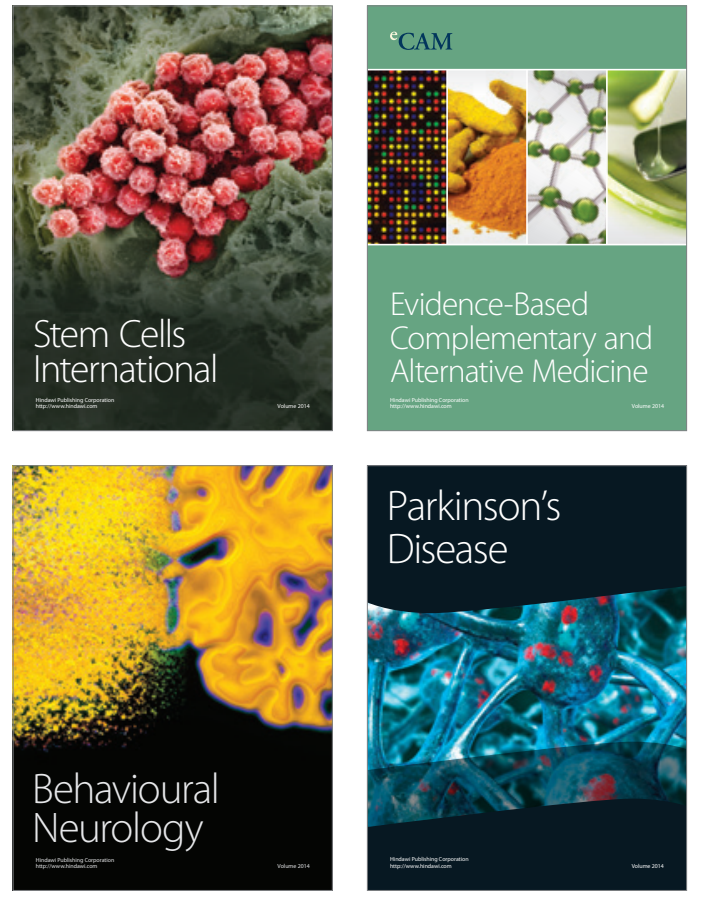
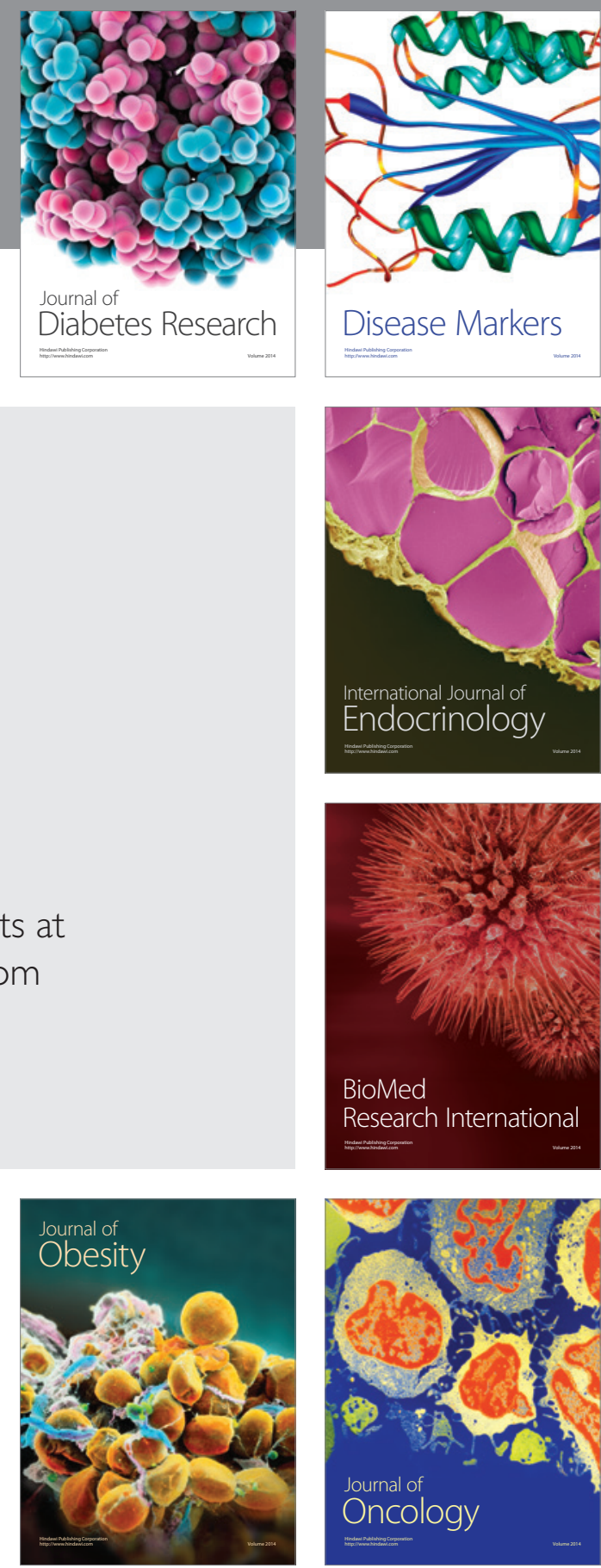

Disease Markers
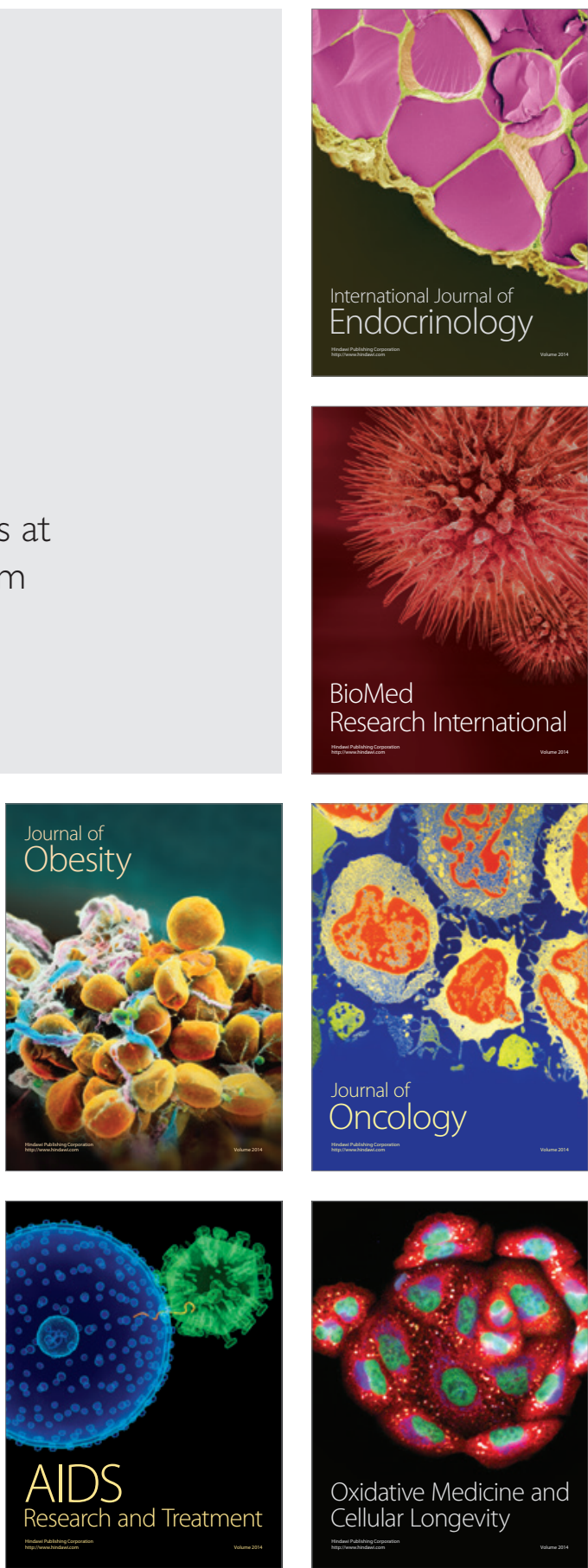